OPEN ACCESS

Edited by:

Bellamkonda K. Kishore,

University of Utah Health Care,

United States

Reviewed by:

Laith Farah Al-rabadi,

University of Utah Hospital,

United States

Alan Pao,

Stanford University, United States

*Correspondence:

Beata Olas

beata.olas@biol.uni.lodz.pl

Specialty section:

This article was submitted to Renal and Epithelial Physiology,

a section of the journal

Frontiers in Physiology

Received: 27 February 2019 Accepted: 06 June 2019

Published: 21 June 2019

Citation:

Woźniak P, Kontek B, Skalski B, Król A, Różański W and Olas B (2019)

Oxidative Stress and Hemostatic

Parameters in Patients With Nephrolithiasis Before and After

Ureteroscopic Lithotripsy.

Front. Physiol. 10:799

doi: 10.3389/fphys.2019.00799

\section{Oxidative Stress and Hemostatic Parameters in Patients With Nephrolithiasis Before and After Ureteroscopic Lithotripsy}

\author{
Paweł Woźniak ${ }^{1}$, Bogdan Kontek ${ }^{2}$, Bartosz Skalski², Anna Król' ${ }^{2}$, Waldemar Różański ${ }^{1}$ \\ and Beata Olas ${ }^{2 *}$ \\ 1 2nd Department of Urology, Medical University of Łódź, Łódź, Poland, ${ }^{2}$ Department of General Biochemistry, Faculty \\ of Biology and Environmental Protection, University of Łódź, Łódź, Poland
}

Purpose: In patients with nephrolithiasis, oxidative stress, especially lipid peroxidation is observed. Moreover, various invasive methods [including extracorporeal shock wave lithotripsy (ESWL)] for treatment of nephrolithiasis may induce not only the oxidative stress, but they may modulate hemostasis. The study was aimed to evaluate the oxidative damages of lipids and proteins in patients with nephrolithiasis (before and after ureteroscopic lithotripsy - URSL). The aim of the present study was also determine selected parameters of hemostasis in these patients.

Methods: 56 patients with nephrolithiasis and 49 healthy participants were included: 30 men and 26 women (for patient group); 27 men and 22 women (for healthy group). We measured the level of selected typical two biomarkers of oxidative modification of lipids [such as the production of thiobarbituric acid reactive substances (TBARS) and isoprostane concentration (8-isoPGF $\left.{ }_{2 \alpha}\right)$ ] and two biomarkers of oxidative damages of proteins (carbonylation and the level of thiol groups) in patients with nephrolithiasis (before and after URSL). The following parameters of hemostasis were measured: blood platelet count, the level of fibrinogen and D-dimer, and coagulation times (the activated partial thromboplastin time (APTT), prothrombin time (PT), and thrombin time (TT) of plasma).

Results: Different levels of plasma lipid peroxidation were observed in patients with nephrolithiasis before URSL and after URSL. However, no such difference in the level of oxidative damage to plasma proteins was observed. In addition, the tested hemostasis parameters were not influenced by the presence of nephrolithiasis, nor by treatment with URSL.

Conclusion: We suggest URSL does not induce the oxidative modifications of plasma proteins and does not change hemostatic parameters in patients with nephrolithiasis.

Keywords: hemostasis, nephrolithiasis, oxidative stress, ureteroscopic lithotripsy, extracorporeal shock wave lithotripsy 


\section{INTRODUCTION}

Oxidative stress causes damage to different biomolecules lipids, proteins and DNA. Moreover, oxidative stress may induce changes elements of hemostasis (including coagulation process and blood platelet activation) in various diseases. In recent years, various studies demonstrated that oxidative stress exists in patients with nephrolithiasis, however, the mechanism(s) of this process is not well known. It has been previously shown that especially lipid peroxidation [measured by different biomarkers: 8-isoprostaglandin $\mathrm{F}_{2}\left(8\right.$-iso $\left.\mathrm{PGF}_{2 \alpha}\right)$ and thiobarbituric acid reactive substances (TBARS)] exists in these patients. Increase of lipid peroxidation was demonstrated in different biological materials (serum, plasma, erythrocytes, and urine) obtained from patients with nephrolithiasis (Khan, 2005, 2012, 2013a,b, 2014; Boonla et al., 2007; Ma et al., 2014; Ceban et al., 2016). In addition, various invasive methods [including extracorporeal shock wave lithotripsy (ESWL)] for treatment of nephrolithiasis may also induce the oxidative stress (Gecit et al., 2012; Garca et al., 2014; Wozniak et al., 2017, 2018) and modulate hemostasis (Wozniak et al., 2017). Modulation of hemostasis was observed 30-240 min after shock wave lithotripsy (SWL) (Hughes et al., 2015) and the day after ESWL (Wozniak et al., 2017).

Ureteroscopic lithotripsy (URSL) is one of the most common operations in urology. Moreover, it is and important that is an effective and safe method for managing ureteral stones (Seitz et al., 2007; Tao et al., 2015). Complications occur in less than $0.1 \%$ of cases (Nuttall et al., 2010). However, the effect of URSL on parameters of oxidative stress and hemostasis in patients with nephrolithiasis has not been studied yet. Thus, the main objective of our experiments was to examine the level of selected typical two biomarkers of oxidative modification of lipids (such as the production of TBARS and isoprostane concentration) and two biomarkers of oxidative damages of proteins (carbonylation and the level of thiol groups) in patients with nephrolithiasis (before and after URSL). Moreover, the aim of the present study was also determine selected parameters of hemostasis (blood platelet count, the level of fibrinogen and D-dimer, and coagulation times (the activated partial thromboplastin time (APTT), prothrombin time (PT), and thrombin time (TT) of plasma) in these patients.

\section{MATERIALS AND METHODS}

\section{Patients and Samples}

The blood samples were collected from 56 patients (30 men and 26 women) whom had been referred to the 2nd Department of Urology, Medical University of Łódź, Poland, for URSL. The exclusion criteria were as follows: the presence of urinary tract infection, other inflammatory or malignant disease, diabetes mellitus, history of heart disease, or chronic kidney disease. Before URSL anatomy of the kidney and excretory path for all patients were investigated by intravenous urography, and no anomalies were found.
A group of 49 healthy individuals (27 men and 22 women) were collected from the hospital from routine controls of health and used as control. They were non-related men and women, that have never been diagnosed with nephrolithiasis or chronic disease and were randomly selected and frequency matched to the cases on age.

The blood and urine samples were collected from the patients before URSL, and 1 day after URSL. The blood samples and the urine samples were taken from patients and healthy participants keeping a balanced diet (meat and vegetables), with similar socio-economic background, using no antioxidant supplementation and any medications (f.e. anti-platelet drugs or anti-inflammatory agents).

Erythrocytes were separated from plasma by centrifugation $(2800 \times g$ for $10 \mathrm{~min})$. Concentration of hemoglobin was determined by the cyanohemoglobin method using Drabkin's reagent. Participants provided also first morning void urine samples $(50-100 \mathrm{ml})$, which was kept on ice and processed within $4 \mathrm{~h}$. Plasma samples obtained from the participants were stored at $-80^{\circ} \mathrm{C}$ within $2 \mathrm{~h}$ of removal.

The protocol was passed by the Committee for Research on Human Subjects of the Medical University of Łódź RNN/101/13/KE. The protocol was passed by the Committee for Research on Human Subjects of the Medical University of Łódź RNN/101/13/KE. The first, participants provided verbal consent to the researchers, and later participants provided written the documents. Authors had access to identifying participant information.

\section{Parameters of Oxidative Stress Measurement of Lipid Peroxidation - The Level of 8-isoPGF $2 \alpha$}

The level of 8-isoPGF $2 \alpha$ was estimated in urine samples from control subjects and from patients using an immunoassay kit (Cayman Chemical) according to the manufacturer's instructions.

\section{Measurement of Lipid Peroxidation - The Level of TBARS}

Samples of plasma or erythrocytes were transferred to an equal volume of cold $20 \%$ (v/v) trichloroacetic acid in $0.6 \mathrm{M} \mathrm{HCl}$ and centrifuged at $1200 \times g$ for $15 \mathrm{~min}$. One volume of clear supernatant was mixed with 0.2 volume of $0.12 \mathrm{M}$ thiobarbituric acid in $0.26 \mathrm{M}$ Tris ( $\mathrm{pH}$ 7.0) immersed in a boiling water bath for $15 \mathrm{~min}$. and then absorbance was measured at $535 \mathrm{~nm}$ (the SPECTROstar Nano Microplate Reader - BMG LABTECH Germany) (Wachowicz, 1984; Bartosz, 2008). The TBARS concentration was calculated using the molar extinction coefficient $\left(\varepsilon=156,000 \mathrm{M}^{-1} \mathrm{~cm}^{-1}\right)$.

\section{Measurement of Protein Carbonylation}

The detection of carbonyl groups in proteins was carried out according to Levine et al. (1990) and Bartosz (2008). The carbonyl group concentration was calculated using a molar extinction coefficient $\left(\varepsilon=22,000 \mathrm{M}^{-1} \mathrm{~cm}^{-1}\right)$, and the level of carbonyl groups was expressed as nmol carbonyl 
3

$(+270 \%, p<0.001)$

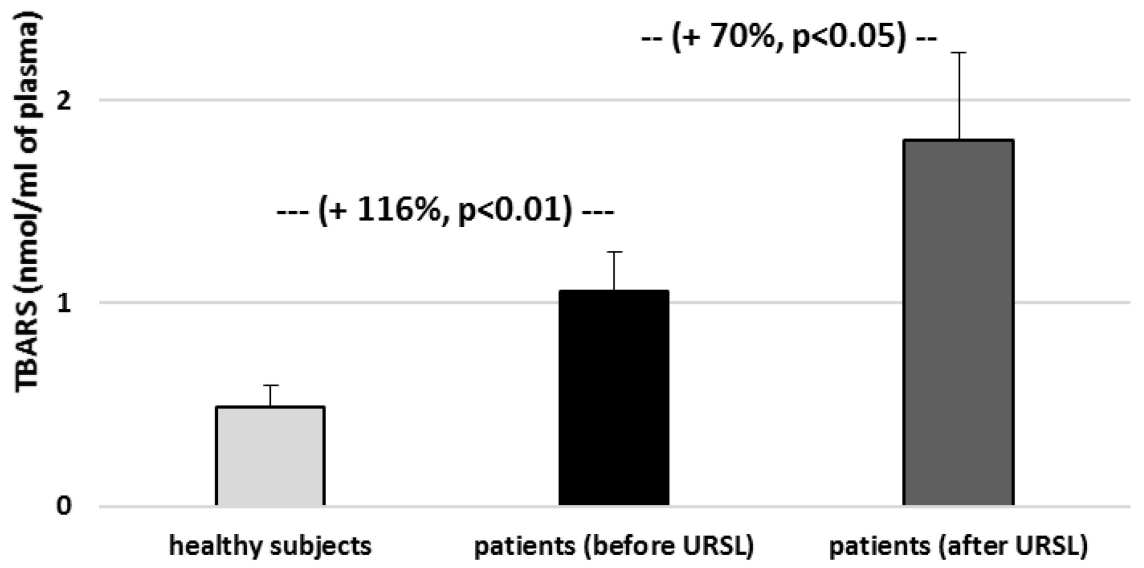

FIGURE 1 | The level of TBARS in plasma in patients with nephrolithiasis (before and after URSL), and in control plasma obtained from healthy volunteers. Results are means $\pm S D$. The statistically analysis was done by Mann-Whitney test (for means $\pm S D$ ).

80

$(+35 \%, p<0.05)$

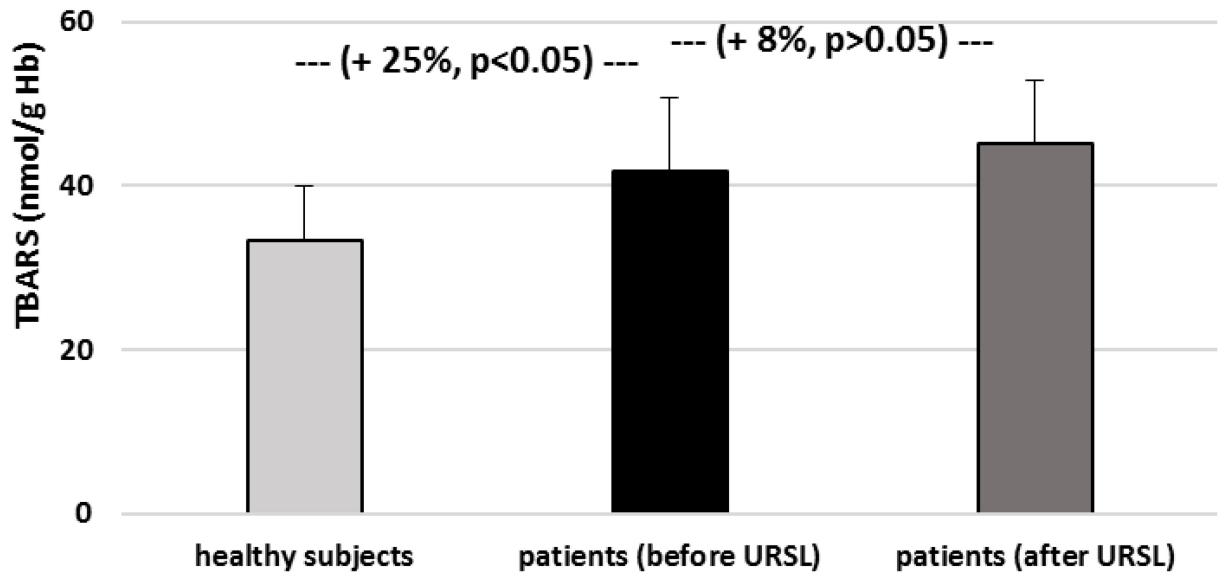

FIGURE 2 | The level of TBARS in erythrocytes in patients with nephrolithiasis (before and after URSL), and in control erythrocytes obtained from healthy volunteers. Results are means \pm SD. The statistically analysis was done by Mann-Whitney test (for means \pm SD).

groups/mg of protein. Carbonyl content was determined by taking the SPECTROstar Nano Microplate Reader - BMG LABTECH Germany.

\section{Measurement of the Level of Thiol Groups}

The thiol group content was measured spectrophotometrically (the SPECTROstar Nano Microplate Reader - BMG LABTECH Germany) by absorbance at $412 \mathrm{~nm}$ with Ellman's reagent: 5,5'-dithio-bis-(2-nitrobenzoic acid). The thiol group concentration was calculated using a molar extinction coefficient $\left(\varepsilon=13,600 \mathrm{M}^{-1} \mathrm{~cm}^{-1}\right.$ ) (Ando and Steiner, 1973a,b;
Bartosz, 2008). The level of thiol groups was expressed as nmol thiol groups/mg of plasma protein.

\section{Parameters of Hemostasis}

The Measurement of Prothrombin Time

The PT (seconds) was determined coagulometrically (BCS XP Healthcare Diagnostics Siemens, Germany) in citrated samples.

\section{The Measurement of Thrombin Time}

The TT (seconds) was determined coagulometrically (BCS XP Healthcare Diagnostics Siemens, Germany) in citrated samples. 


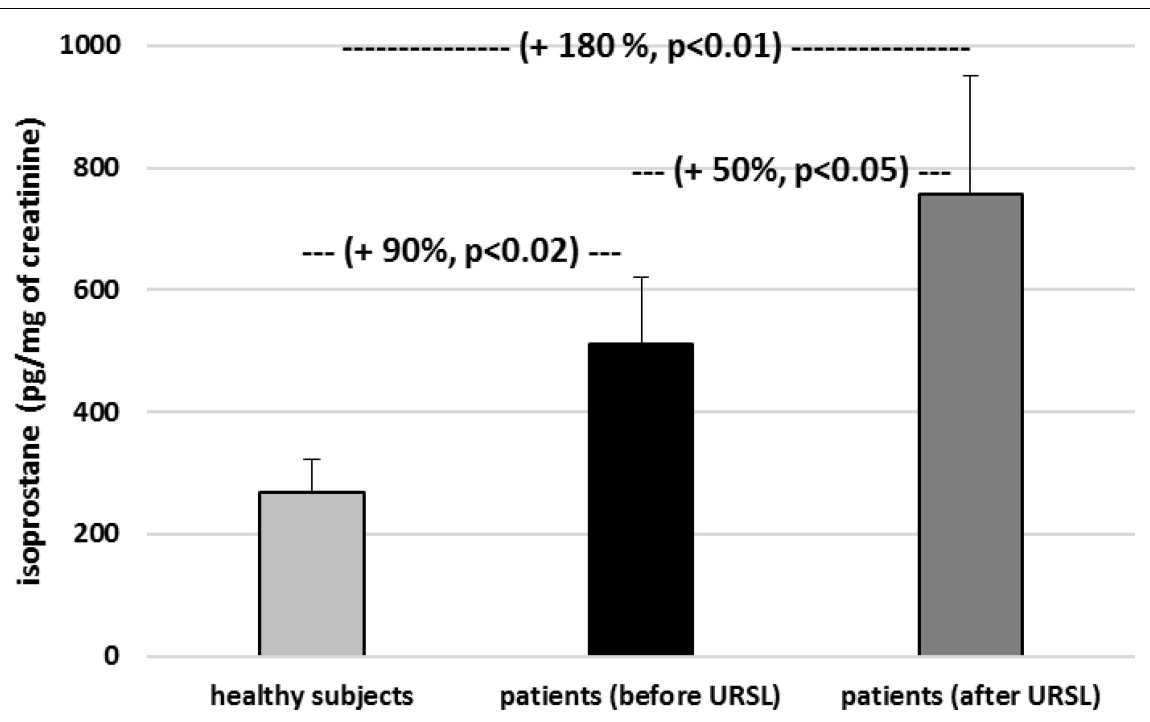

FIGURE 3| The level of 8-isoPGF $2 \alpha$ in urine in patients with nephrolithiasis (before and after URSL), and in control urine obtained from healthy volunteers. Results are means $\pm \mathrm{SD}$. The statistically analysis was done by Mann-Whitney test (for means $\pm \mathrm{SD}$ ).

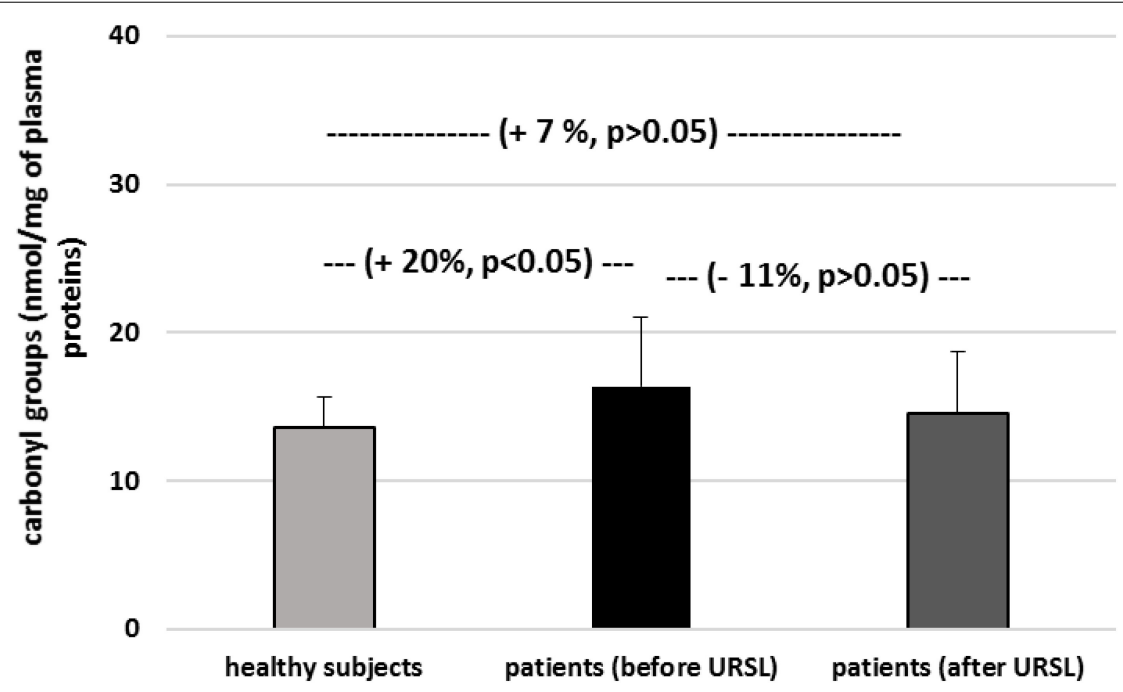

FIGURE 4 | The level of carbonyl groups in plasma proteins in patients with nephrolithiasis (before and after URSL), and in control plasma proteins obtained from healthy volunteers. Results are means \pm SD. The statistically analysis was done by Mann-Whitney test (for means \pm SD).

\section{The Measurement of APTT}

The APTT (seconds) was determined coagulometrically (BCS XP Healthcare Diagnostics Siemens, Germany) in citrated samples.

\section{The Measurement of Blood Platelet Concentration}

Blood platelet count was performed using an automated cell counter (Sysmex XN-2000, Sysmex, Japan) in citrated samples. The platelets were measured in units $\times 10^{9} / \mathrm{l}$.

\section{The Measurement of Fibrinogen}

Fibrinogen ( $\mathrm{g} / \mathrm{l})$ concentration (in citrated samples) was measured using an analyzer (BCS XP Healthcare Diagnostics Siemens, Germany).

\section{The Measurement of D-Dimer}

D-dimer $(\mathrm{ng} / \mathrm{ml})$ concentration was determined by an analyser (BCS XP Healthcare Diagnostics Siemens, Germany) in citrated samples.

\section{Statistical Analysis}

The statistical analysis was done by several tests. All the values in this study were expressed as mean \pm SD. In order to eliminate uncertain data, Q-Dixon test was performed. Since levels of biomarkers of oxidative stress in studied material did not show normal distribution (Kolmogorov-Smirnov test) the non-parametrical statistical test (Mann-Whitney $U$ test) was applied. Reported 


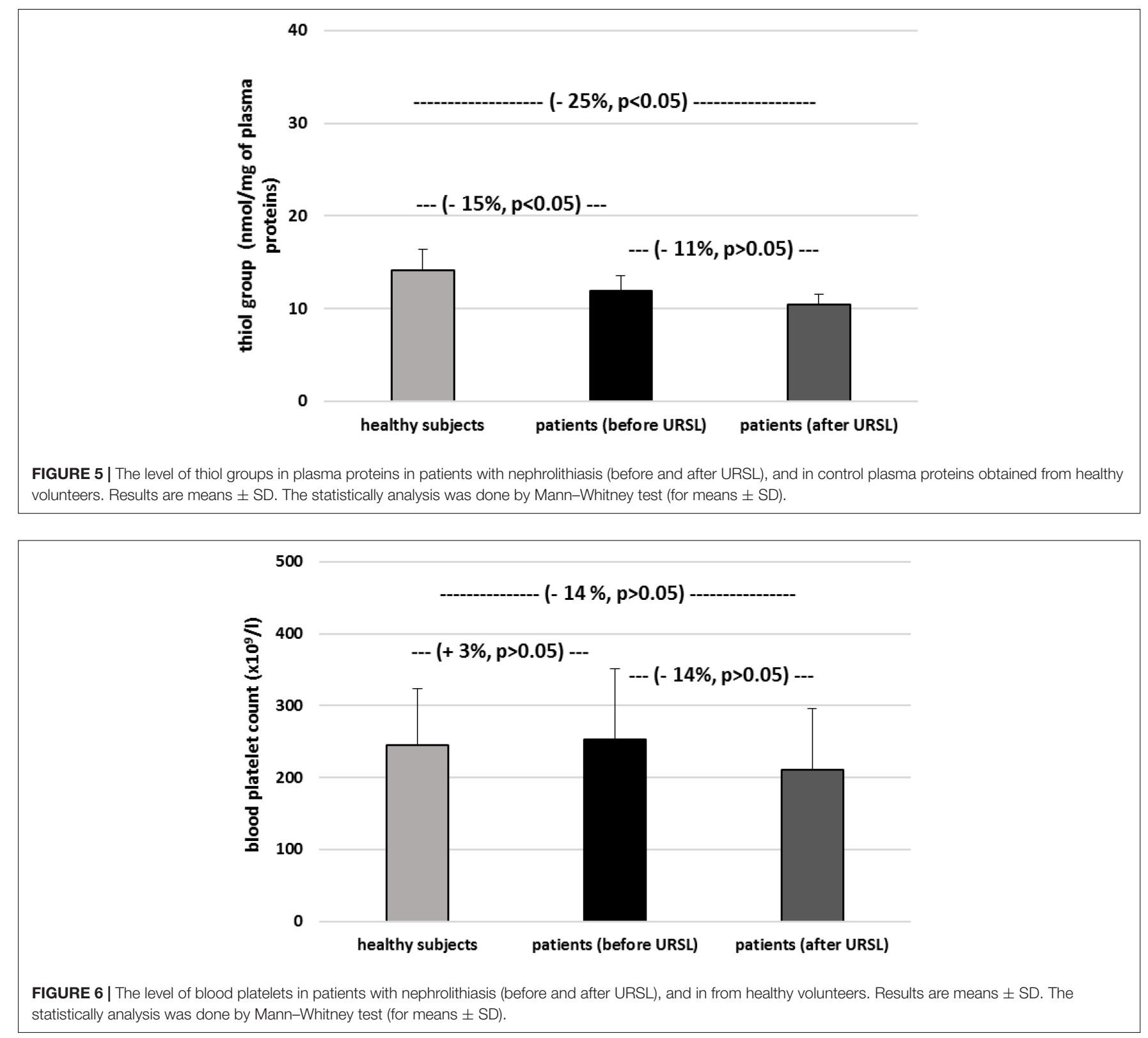

$p$-values were two-sided. All analyses were completed using STATISTICA 12.

\section{RESULTS}

As demonstrated on Figure 1, the TBARS level in plasma was significantly increase in patients with nephrolithiasis (before URSL) than in control. In plasma samples from patients with nephrolithiasis (before URSL), the level of TBARS was increase by about $116 \%$ compare to control group (Figure 1). The concentration of TBARS in plasma from patients with nephrolithiasis (after URSL) was also higher than in patients with nephrolithiasis (before URSL) and higher than in healthy volunteers (Figure 1). In addition, the concentration of TBARS in erythrocytes in patients with nephrolithiasis (before URSL and after URSL) differed markedly from healthy volunteers (Figure 2). The TBARS content in erythrocytes in patients with nephrolithiasis (before URSL and after URSL) was significantly higher than in controls [and increase - about 25\% (before URSL), and about 35\% (after URSL)] (Figure 2). The level of TBARS in erythrocytes from patients after URSL was also changed, compared with patients before URSL, however, it was not statistically significant (Figure 2).

Other experiments showed that the concentration of isoprostane in urine from patients (before and after URSL) was found to be higher than the concentration of isoprostane in urine obtained from healthy volunteers (Figure 3). The concentration of isoprostane in urine from patients (after URSL) was also higher than in patients (before URSL) (Figure 3). 


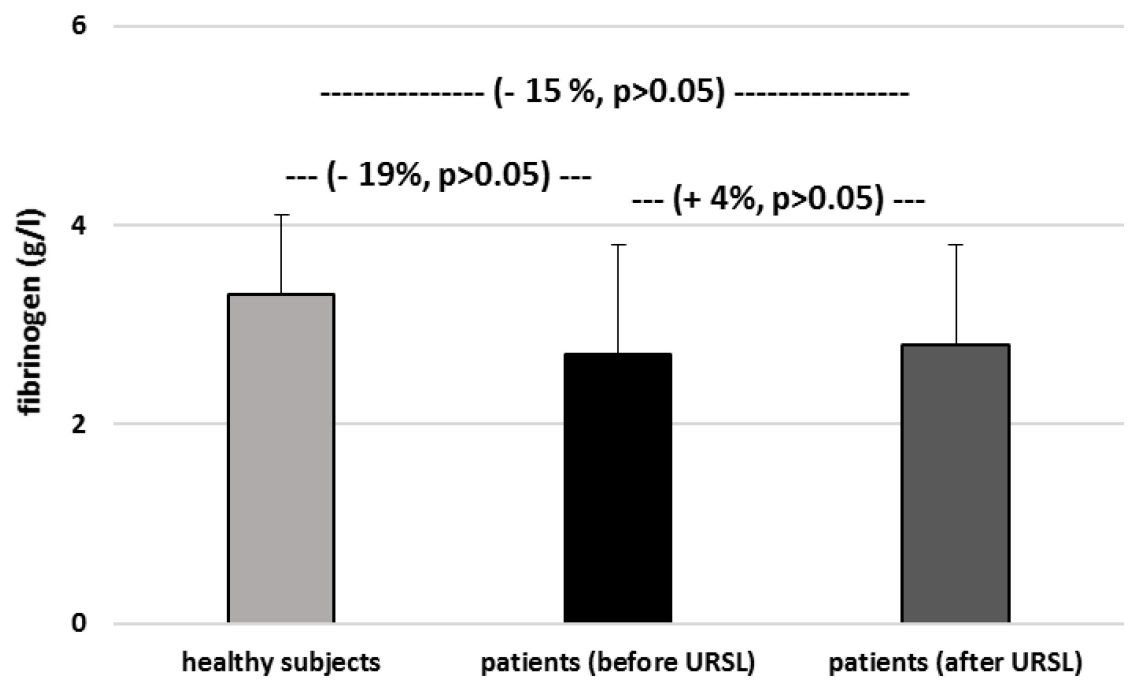

FIGURE 7 | The level of fibrinogen in plasma from patients with nephrolithiasis (before and after URSL), and in control plasma obtained from healthy volunteers. Results are means $\pm \mathrm{SD}$. The statistically analysis was done by Mann-Whitney test (for means \pm SD).

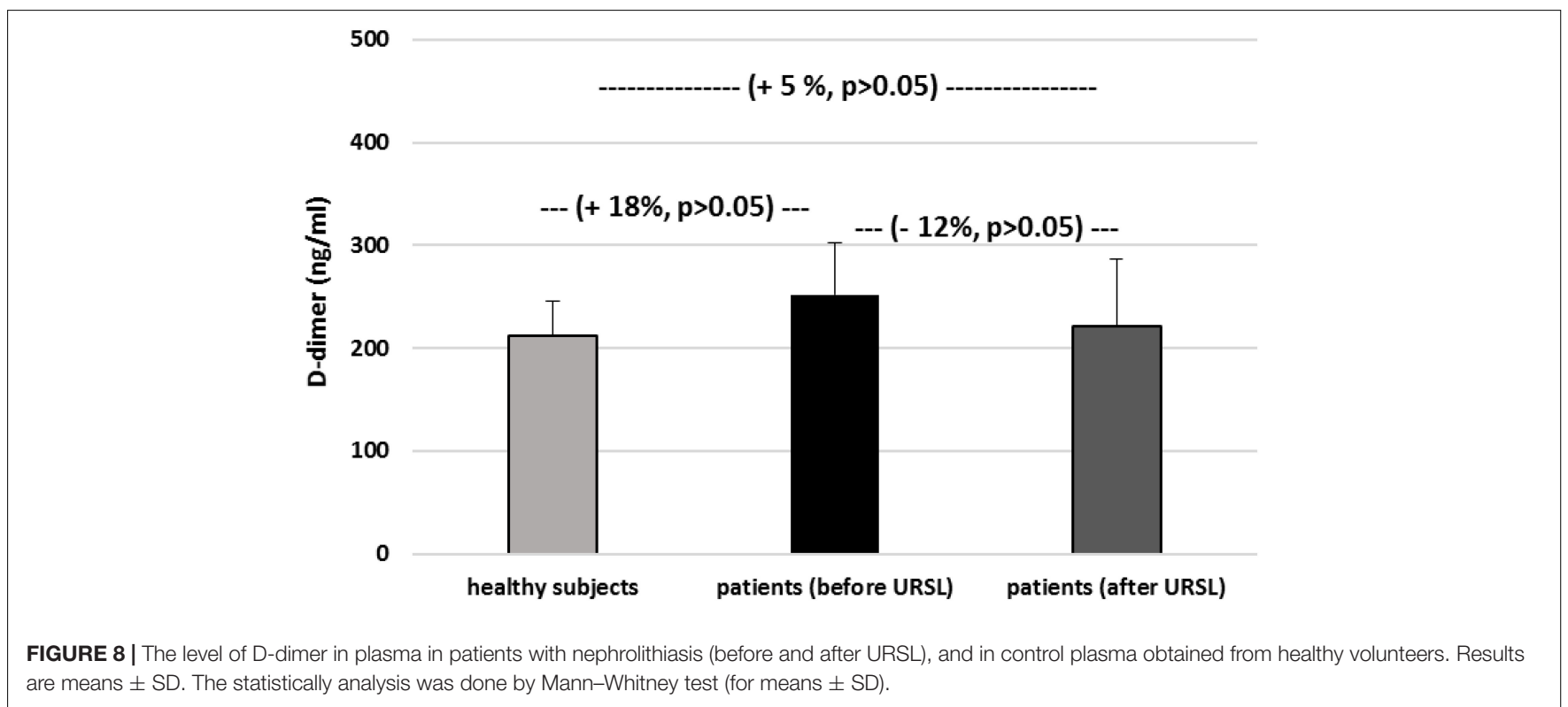

The level of carbonyl groups in plasma proteins from patients with nephrolithiasis (before and after URSL) was found to be higher than the level of carbonyl groups in plasma proteins obtained from healthy volunteers (Figure 4). On the other hand, the level of carbonyl groups in proteins from patients (after URSL) was lower than in proteins from patients (before URSL) (Figure 4). However, this change (in the concentration of carbonyl groups) was not statistically significant (Figure 4). Figure 5 demonstrates that the level of thiol groups in proteins from patients (after URSL) was slightly lower than in proteins from patients (before URSL), but not statistically significant.

Figures 6-9 demonstrate parameters of hemostasis in patients with nephrolithiasis before and after URSL. For example, blood platelet counts were (about 14\%) lower in patients after URSL than in healthy subjects or in patients before URSL, but these changes were not statistically significant (Figure 6). Neither, fibrinogen, D-dimer and coagulation times (APTT, PT, and TT) were influenced by the presence of nephrolithiasis, nor by treatment with URSL (Figures 7-9).

Moreover, we did not observe the difference in concentrations of biomarkers of oxidative stress between the group of men and the group of women.

\section{DISCUSSION}

Lipids and proteins are major targets for oxidants. Oxidative stress itself is known to play a role in nephrolithiasis through 


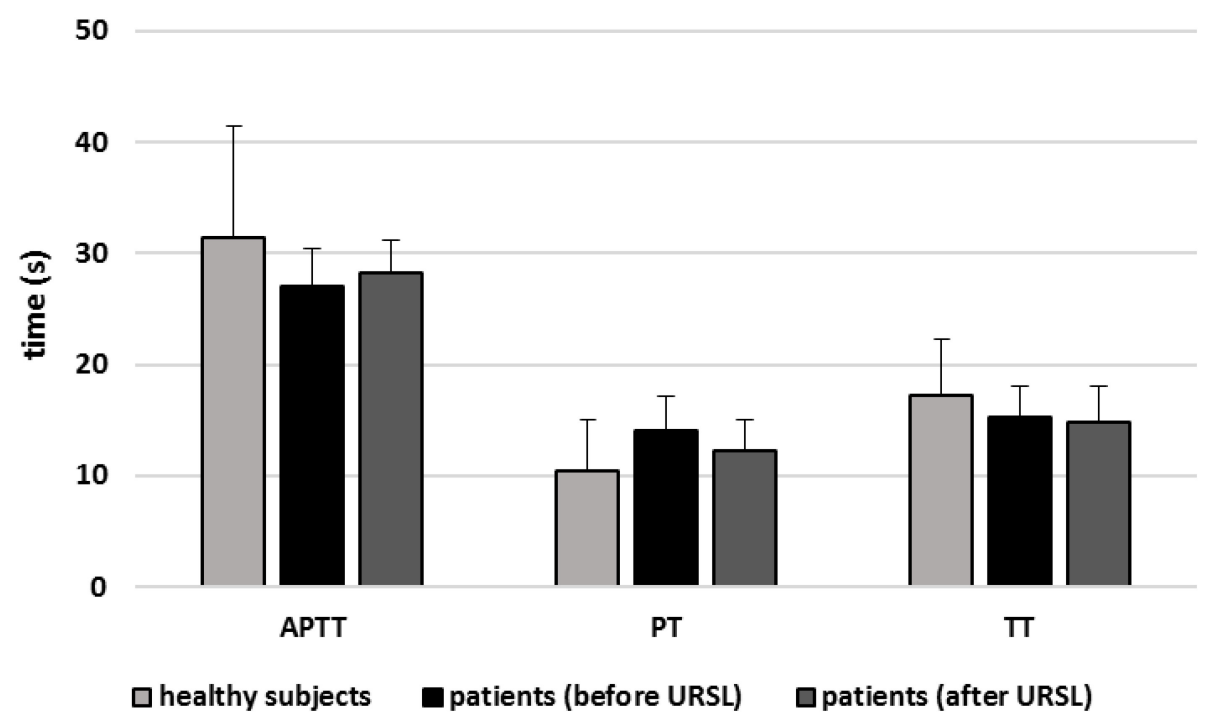

FIGURE 9 | The activated partial thromboplastin time (APTT), prothrombin time (PT), and thrombin time (TT) of plasma in patients with nephrolithiasis (before and after URSL), and in control plasma obtained from healthy volunteers. Results are given as means \pm SD. The statistical analysis was performed using the

Mann-Whitney test (for all times: $p>0.05$ - patients [(before/after URSL] versus healthy subjects; $p>0.05$ patients [after ESWL] versus patients [(before URSL]).

the action of free radicals, which are believed to initiate the inflammation process and induce renal cellular injury (Khan, 2005, 2012, 2013a,b, 2014; Boonla et al., 2007; Ma et al., 2014; Ceban et al., 2016). In addition, increased oxidative stress may also be correlated with kidney stone development (Gaspar et al., 2010). For example, oxidative stress has been reported in the erythrocytes of patients with calcium oxalate stones, resulting in renal tubular damage and increases in stone size (Ma et al., 2014). Other studies suggest that urinary levels of oxalate and citric oxide may be correlated with lipid peroxidation (Huang et al., 2003). The present study by using different biomarkers of oxidative stress - damages of lipids, including the change of TBARS (in plasma and erythrocytes) and the change of isoprostane level in urine provides evidence that in patients with nephrolithiasis, oxidative stress - lipid peroxidation occurs. It should be underlined that in our present experiments tested samples from patients were taken before and after URSL. It is very important, because various drugs, which are used during treatment (i.e., surgery) may induce oxidative stress in different tissues, blood cells and plasma (Wang et al., 2017). However, Ceban et al. (2016) studied oxidant and antioxidant status in the blood of patients with complicated urolithiasis pre- and post-surgery. Oxidant and antioxidant status was measured also by various parameters, including the level of thiol groups and activity of different antioxidant enzymes (i.e., glutathione dismutase and glutathione reductase). Experiments of these authors demonstrated that the surgical treatment of complicated urolithiasis leads a decrease of the oxidative stress and an increase in potential of antioxidant status. On the other hand, in vitro and in vivo experiments show that increased oxidative stress is associated with kidney stone development (Khan, 2005, 2012, 2013a,b, 2014; Boonla et al., 2007;
Ma et al., 2014; Ceban et al., 2016). Our earlier results indicate that ESWL also induces the oxidative stress (measured by the level of carbonyl groups in plasma proteins in patients with nephrolithiasis) and modulates hemostasis in these patients (Wozniak et al., 2017). A key novel finding of this experiment is that patients with nephrolithiasis undergoing other method for treatment - URSL, experience an increase in lipid peroxidation (measured by two typical biomarkers: TBARS and isoprostanes), as compared to healthy volunteers. Moreover, changes in lipid peroxidation were found between patients before URSL and patients who had completed treatment.

Hemostasis is thought to be modulated by oxidative stress (Nowak et al., 2010). However, in the present study, no changes in oxidative damage to plasma proteins, indicated by the levels of thiol groups and carbonyl groups, were observed in the nephrolithiasis patients before URSL and after URSL. In addition, a significant novel finding is that URSL does not appear to induce changes in hemostasis, measured by various typical parameters, including blood platelet count, fibrinogen concentration and coagulation times in these patients. The only possible explanation for this lack of observed change was that no oxidative damage had occurred to the plasma proteins. Hughes et al. (2015) also report that treatment of solitary kidney stones by SWL does not appear to influence the biochemical parameters of hemostasis, measured by coagulation times (APTT and PT).

This present study is the first to examine the effect of URSL on parameters of oxidative stress and hemostasis in patients with nephrolithiasis. Our findings indicate that URSL does not induce any oxidative modification in plasma proteins nor does it change the hemostatic parameters in these patients. However, the differences in levels of oxidative stress biomarkers are small, but they are statistically significant. Moreover, it is not 
clear whether these statistical changes are clinically significant. Therefore, further experiments based on larger groups of patients are needed to more precisely determine its influence on oxidative stress and hemostasis.

\section{DATA AVAILABILITY}

The raw data supporting the conclusions of this manuscript will be made available by the authors, without undue reservation, to any qualified researcher.

\section{ETHICS STATEMENT}

The protocol was passed by the Committee for Research on Human Subjects of the Medical University of Łódź

\section{REFERENCES}

Ando, Y., and Steiner, M. (1973a). Sulphydryl and disulphide groups of platelet membranes: determination of disulphide groups. Biochim. Biophys. Acta 311, 26-37.

Ando, Y., and Steiner, M. (1973b). Sulphydryl and disulphide groups of platelet membranes: determination of sulphydryl groups. Biochim. Biophys. Acta 311, 38-44.

Bartosz, G. (2008). Druga twarz tlenu. Warszawa: PWN. 7, 99-120.

Boonla, C., Wunsuwan, R., Tungsanga, K., and Tosukhowong, P. (2007). Urinary 8-hydroxydeoxyguanosie is elevated in patients with nephrolithiasis. Urol. Res. 35, 185-191. doi: 10.1007/s00240-007-0098-0

Ceban, E., Banov, P., Galescu, A., and Botnari, V. (2016). Oxidative stress and antioxidant status in patients with complicated urolithiasis. J. Med. Life 9, 259-262.

Garca, M. F., Kavak, S., Gecit, I., Meral, I., Demir, H., Turan, M., et al. (2014). Effects of shock waves on oxidative stress in parotid gland of rat. Toxicol. Ind. Health 30, 454-458. doi: 10.1177/0748233712457452

Gaspar, S., Niculite, C., Cucu, D., and Marcu, I. (2010). Effect of calcium oxalate on renal cells as revealed by real-time measurement of extracellular oxidative burst. Biosens. Bioelectron. 25, 1729-1734. doi: 10.1016/j.bios.2009.12.013

Gecit, I., Kavak, S., Meral, I., Demir, H., and Ceylan, K. (2012). Effects of shock waves on oxidative stress and some tace element levels of rat liver and diaphragm muscles. Cell Biochem. Funct. 30, 303-308. doi: 10.1002/cbf.2802

Huang, H. S., Ma, M. C., Chen, C. F., and Chen, J. (2003). Lipid peroxidation and its correlations with urinary levels of oxalate, citric acid, and osteopontin in patients with renal calcium oxalate stones. Urology 62, 1123-1128. doi: 10.1016/s0090-4295(03)00764-7

Hughes, S. F., Thomas-Wright, S. J., Banwell, J., Williams, R., Moyes, A. J., Mushtaq, S., et al. (2015). A pilot study to evaluate haemostatic function, following shock wave lithiotripsy (SWL) for the treatment of solitary kidney stones. PLoS One 10:e0125840. doi: 10.1371/journal.pone.0125840

Khan, S. R. (2005). Hyperoxaluria-induced oxidative stress and antioxidants for renal protection. Urol. Res. 33, 349-357. doi: 10.1007/s00240-005-0492-4

Khan, S. R. (2012). Is oxidative stress, a link between nephrolithiasis and obesity, hypertension, diabetes, chronic kidney disease, metabolic syndrome? Urol. Res. 40, 95-112. doi: 10.1007/s00240-011-0448-9

Khan, S. R. (2013a). Reactive oxygen species as the molecular modulators of calcium oxalate kidney stone formation: evidence from clinical and experimental investigations. J. Urol. 189, 803-811. doi: 10.1016/j.juro.2012. 05.078

Khan, S. R. (2013b). Stress oxidative: nephrolithiasis and chronic kidney diseases. Minerva Med. 104, 23-30.

Khan, S. R. (2014). Reactive oxygen species, inflammation and calcium oxalate nephrolithiasis. Transl. Androl. Urol. 3, 256-276.
RNN/101/13/KE. The first, participants provided verbal consent to the researchers, and later participants provided written the documents. Authors had access to identifying participant information.

\section{AUTHOR CONTRIBUTIONS}

All authors listed have made a substantial, direct and intellectual contribution to the work, and approved it for publication.

\section{FUNDING}

This work was supported by grant B1711000000044.01 from the University of Łódź.

Levine, R. L., Garland, D., Oliver, C. N., Amici, A., Climent, I., Lenz, A. G., et al. (1990). Determination of carbonyl content in oxidatively modified proteins. Methods Enzymol. 186, 464-478. doi: 10.1016/0076-6879(90)86 $141-\mathrm{h}$

Ma, M.-C., Chen, Y.-S., and Huang, H.-S. (2014). Erythrocyte oxidative stress in patients with calcium oxalate stones correlates with stone size and renal tubular damage. Urology 8, 1-9. doi: 10.1016/j.urology.2013.09.050

Nowak, P., Olas, B., and Wachowicz, B. (2010). Oxidative stress in haemostasis. Postepy Biochem. 56, 239-247.

Nuttall, M. C., Abbaraju, J., Dickinson, I. K., and Sriprasad, S. (2010). A review of studies reporting on complications of upper urinary tract stone ablation using the holmium: YAG laser. Br. J. Med. Surg. Urol. 3, 151-159. doi: 10.1016/j. bjmsu.2010.01.008

Seitz, C., Tanovic, E., Kikic, Z., and Fajkovic, H. (2007). Impact of stone size, locatiom, composition, impaction, and hydronephrosis on the efficacy of holmium: YAG-laser ureterolithotripsy. Eur. Urol. 52, 1751-1757.

Tao, W., Cai, C. J., Sun, C. Y., Xue, B. X., and Shan, Y. X. (2015). Subcapsular renal hematoma after ureteroscopy with holmium:yttrium-aluminum-garnet laser lithotripsy. Lasers Med. Sci. 30, 1527-1532. doi: 10.1007/s10103-015-1760-3

Wachowicz, B. (1984). Adenine nucleotides in thrombocytes of birds. Cell. Biochem. Funct. 2, 167-170. doi: 10.1002/cbf.290020310

Wang, Y., Zhong, B., Yang, X., Wang, G., Hou, P., and Meng, J. (2017). Comparison of the efficacy and safety of URSL, RPLU, and MPCNL for treatment of large upper impacted ureteral stones: a randomized controlled trial. BMC Urol. 17:50. doi: 10.1186/s12894-017-0236-0

Wozniak, P., Kontek, B., Różański, W., and Olas, B. (2017). Evaluation of hemostasis parameters and the role of the oxidative damage to plasma proteins in the modulation of hemostasis in patients with nephrolithiasis before and after extracorporeal shock wave lithotripsy. PLoS One 10:e0185157. doi: 10.1371/ journal.pone. 0185157

Wozniak, P., Kontek, B., Różański, W., and Olas, B. (2018). The lipid peroxidation in patients with nephrolithiasis before and after extracorporeal shock wave lithotripsy. Future Med. Chem. 10, 2685-2693. doi: 10.4155/fmc-2018-0149

Conflict of Interest Statement: The authors declare that the research was conducted in the absence of any commercial or financial relationships that could be construed as a potential conflict of interest.

Copyright (® 2019 Woźniak, Kontek, Skalski, Król, Różański and Olas. This is an open-access article distributed under the terms of the Creative Commons Attribution License (CC BY). The use, distribution or reproduction in other forums is permitted, provided the original author(s) and the copyright owner(s) are credited and that the original publication in this journal is cited, in accordance with accepted academic practice. No use, distribution or reproduction is permitted which does not comply with these terms. 